\title{
Metodología para la Revisión y Actualización de un Diseño Curricular de una Carrera Universitaria Incorporando Conceptos de Aprendizaje Basado en Competencias
}

\author{
Gabriel A. Icarte ${ }^{(1)}$ y Hugo A. Labate ${ }^{(2)}$ \\ (1) Facultad de Ingeniería y Arquitectura. Universidade Arturo Pra. Havia. Arturo Pra № 2120. Iquique, \\ Chile. (e-mail: gabriel.icarte@unap.cl) \\ (2) Facultad de Ciencias Sociales. Universidad Nacional de Lomas de Zamora. Trinidad 1031 (B1667GWU) \\ Tortuguitas Provincia de Buenos Aires - Argentina. (e-mail: hugo.labate@gmail.com)
}

Recibido Jul. 6, 2015; Aceptado Ago. 11, 2015; Versión final Oct. 10, 2015, Publicado Abr. 2016

\begin{abstract}
Resumen
Este artículo presenta una experiencia en la revisión del diseño curricular incorporando los conceptos de aprendizaje basado en competencias. Se señalan las razones del porqué de esta revisión y plantea una propuesta metodológica compuesta de cinco etapas. El proceso de revisión del diseño curricular en una carrera universitaria es una tarea ardua y compleja que se realiza en diversas etapas y finaliza cuando los actores consideran que se ha alcanzado un producto que satisface los objetivos iniciales. Las cinco etapas de la propuesta son: (i) procesamiento pedagógico de las competencias establecidas en el perfil; (ii) elaboración de una secuencia de progresión de las competencias; (iii) análisis sobre el aporte de las asignaturas al desarrollo de las competencias; (iv) actualización de los programas y de la malla curricular; y (v) producción de pruebas de logros. Los principales resultados obtenidos al aplicar la metodología fueron la modificación de los programas de asignaturas y pruebas de logros, la incorporación de mapas visuales que permiten observar el aporte de las asignaturas al desarrollo de las competencias y la visualización del desarrollo de las competencias durante el plan de formación curricular.
\end{abstract}

\section{Methodology for Reviewing and Updating a Curriculum Design of a University Career Incorporating the Concept of Competency-based Learning}

\begin{abstract}
This article presents a review of the experience gained after revising and updating the design of an academic curriculum incorporating the concept of competency-based learning. The reasons to revise the curriculum are discussed and a methodological proposal composed by five stages is presented. The modification of the academic curriculum of a university career is a difficult and complex task that is performed in different stages and the process ends when all the professionals involved believe that a real product, that satisfies the initial objectives, has been reached. The five steps of the proposal are: (i) pedagogical processing of the competencies established in the students profile; (ii) establishing a progress map for sequencing the competencies; (iii) analysis about the contribution of subjects to the development of each of the competencies; (iv) updating of the programs included in the syllabus grid; and (v) the elaboration of achievement tests. The main results obtained in applying the methodology were the modification of the original subject syllabuses, incorporation of visual maps for observing the contribution of the subjects to the development of competencies, and the visualization of the development of competencies during the training curriculum plan.
\end{abstract}




\section{INTRODUCCIÓN}

Los procesos de actualización curricular de las carreras universitarias suelen llevarse a cabo en un marco de tensión entre los tiempos que se precisan para el debate de cátedras y con los representantes del campo profesional, que confluyen en la elaboración deductiva del perfil de egreso y posteriormente de la malla curricular, frente a los tiempos administrativos que demandan lograr una actualización de la carrera dentro de plazos acotados, para poder dimensionar los costos de las reformas y formalizar la comunicación a los estudiantes que deben inscribirse en la carrera renovada. Como resultado, las mallas curriculares que se elaboran son productos que apuntan al logro de unas competencias establecidas previamente, pero que en ocasiones no están todavía del todo "maduras" en términos de su coherencia con el perfil de egreso (que la encuadra) y los programas de asignaturas (que se desprenden de ella).

En el marco del proceso de rediseño curricular definido según la Universidad Arturo Prat, la Dirección de la Carrera de Ingeniería Civil en Computación e Informática de esa institución se propuso verificar la coherencia y consistencia de su malla curricular elaborada en 2012, a través del diseño de una metodología y su posterior aplicación para evaluar si el currículo de la carrera responde al cumplimiento del perfil de egreso establecido. Lo anterior forma parte de la estrategia institucional de aseguramiento de calidad de los procesos de aprendizaje.

El punto de partida estaba caracterizado por un desfase entre el currículo renovado y el aporte de las asignaturas al desarrollo de las competencias, puesto que en cada asignatura, las competencias a las cuales la asignatura aportaría para su desarrollo, fueron determinadas por cada profesor, estableciendo un desarrollo no equilibrado en cada una de las competencias. Esta constatación promovió un proceso de revisión y modificación de los programas para que en su conjunto desarrollaran las competencias señaladas en el perfil de manera balanceada.

\section{Aseguramiento de la calidad}

El aseguramiento de la calidad se define como el conjunto de acciones planificadas y sistemáticas, implementadas bajo un Sistema de Calidad, que son necesarias para proporcionar la confianza adecuada de que un producto satisfará los requisitos dados sobre la calidad (ISO 9000:2000). En el ámbito de educación superior, las universidades deben hacerse cargo de los procesos que permitan el aseguramiento de la calidad en diversos aspectos, tales como los proceso de enseñanza-aprendizaje, los servicios prestados por la institución, la gestión interna, el cumplimiento de las normativas legales, entre otros. Para lo anterior existen diversas entidades interesadas en la calidad que entregan las instituciones de educación superior. En el caso de Chile, algunos ejemplos de estas entidades son la División de Educación Superior del Ministerio de Educación, a cargo de asegurar el cumplimiento de las leyes, la Comisión Nacional de Acreditación, a cargo de gestionar la acreditación de las instituciones, las Agencias Acreditadoras a cargo de la acreditación de las carreras, entre otras.

En el contexto de asegurar la calidad de los programas/carreras, las instituciones deben definir prácticas de control, mejoramiento y garantía de la calidad a los diversos componentes de los programas/carreras (planificación, funcionamiento y mecanismo de control de los resultados, planes de formación, etc.) y deben ser de responsabilidad de una entidad que está estrechamente relacionada con la administración del programa/carrera, como por ejemplo el director de carrera o figura equivalente, e incluye las actividades de aseguramiento de la calidad, lo que significa como mínimo evaluar la consistencia entre el currículo, la enseñanza y el logro del perfil de egreso como punto de partida de un ciclo de mejora continua de la oferta curricular de la carrera, que contemple las definiciones marco de las políticas orientadoras del currículo de formación y las estrategias institucionales adecuadas y pertinentes a las metas previstas.

Según Lemaitre y Zenteno (2012), la institucionalización en las universidades del aseguramiento de la calidad ha incorporado modelos de gestión y evaluación que operan sobre el espacio discrecional que típicamente tenían los académicos en modelos curriculares anteriores, asociado a una determinada concepción de "libertad de cátedra" (Madrid, 2013), generando tensiones que deben resolverse en procesos de construcción colectiva de significados. Por otra parte, el aseguramiento de la calidad lleva consigo un aumento de la carga burocrática al requerir un conjunto de procesos y procedimientos formales, llevados a cabo por cuerpos técnicos responsables del programa/carrera.

Una manifestación del aseguramiento de la calidad universitaria está dada por los procesos de revisión del currículo, los cuales pueden originarse en una actualización requerida por necesidades tales como mejorar el proceso de control, incluir cambios producidos en la disciplina profesional y/o incluir cambios en las estrategias de enseñanza. Si el nivel de cambios al currículo es leve, puede ser que una actualización a los programas de asignaturas sea suficiente; pero si los cambios son profundos, será necesario realizar un rediseño del currículo. 


\section{Currículo por competencias}

Sutcliffe et al. (2005) señalan que un currículo basado en competencias se materializa en un conjunto de asignaturas que tienen como objetivos desarrollar en el estudiante una serie de capacidades solicitadas por la sociedad en la que el estudiante se desenvolverá y cuyo desarrollo puede ser demostrado. Este enfoque tiene una serie de ventajas importantes para la educación superior, en particular en aquellas carreras como las ingenierías que tienen un compromiso formativo centrado en el dominio de procedimientos. Lo anterior se debe a que el enfoque de competencias permite expresar mejor las capacidades que deben disponer los egresados al momento de completar sus estudios. De esta manera se facilita el proceso de transición entre el término de los estudios y la incorporación al mercado laboral, en la medida en que los empleadores y los propios egresados tienen mayor información respecto de lo que éstos últimos son capaces de hacer, bajo la garantía de la institución formadora. Según Tobón (2004), en los procesos académicos el enfoque de competencias permite el aseguramiento de la calidad en la medida que es un conjunto de herramientas conceptuales y metodológicas capaces de generar evidencias y controlar la calidad de los procesos de formación. Según Argüelles y Gonczi (2000) este enfoque para el diseño curricular en el nivel universitario es parte del movimiento hacia una Educación Basada en Competencias (CBE por su sigla en inglés) y encuentra sus orígenes en estudios desarrollados por el CINTERFOR de la Organización Internacional del Trabajo en la década del 60. Las instituciones de formación superior comienzan a aplicar este enfoque formativo a partir de los acuerdos de Bolonia (1999) en que varios países se comprometen a asegurar estándares de comparabilidad entre las carreras universitarias en Europa, y se define que estos estándares se asocien a cualificaciones basadas en resultados de aprendizaje amplios, lo que implica revisar la dimensión curricular y reorientar las prácticas de evaluación para llevarlas de un modelo centrado en los contenidos hacia un modelo centrado en los resultados esperados.

El desafío para las universidades, en este marco, reside en rediseñar sus matrices formativas alrededor de las competencias de salida más que alrededor de las tradicionales asignaturas. Sudsomboon (2007) plantea que los cambios principales que esto entraña son 1) pensar en competencias en vez de objetivos, 2) pensar en resultados más que en contenidos, 3) basar las actividades del estudiante sobre desempeños observables antes que en pruebas escritas, 4) desarrollar actividades de enseñanza centradas en el estudiante y 5) enfatizar la evaluación de proceso. Estos cambios deben ser acordados entre los docentes y planteados explícitamente en el nivel curricular que constituye la planificación de los cursos o módulos de aprendizaje.

En este contexto, las instituciones de educación superior que han optado por modelos basados en competencias se han encontrado con diversas dificultades para llevar a la práctica el modelo curricular, entre ellas la evaluación de los aprendizajes acumulativos o longitudinales asociados a las competencias, y la integración al currículo de la formación en competencias genéricas o transversales (CINDA, 2009), por lo que para cumplir con el formativo asumido se requiere de acciones que tiendan a solucionar estas dificultades, desde una perspectiva de aseguramiento de la calidad.

\section{Proceso de rediseño curricular}

Como se mencionó anteriormente, si la modificación a un currículo va más allá de una actualización de contenidos, y si además el currículo está basado en competencias, el proceso de rediseño puede ser bastante complejo. Uno de los problemas más comunes en los rediseños curriculares que se orientan al logro de competencias lo constituye el divorcio que se establece entre las competencias formuladas en el perfil de egreso y las propuestas de enseñanza ofrecidas por los profesores en cada asignatura (Beneitone et Al., 2007), por lo que la gestión académica debe lograr un trabajo consciente y reflexivo de articulación entre competencias y matrices curriculares (nivel macrocurricular); posteriormente, habrá que ajustar el programa de cada unidad de trabajo-aprendizaje (asignaturas, cursos o módulos) en torno a alguna(s) de la(s) competencia(s) declarada en el perfil de egreso, prestando especial atención a la relación entre el nivel de logro esperado para un determinado momento de la carrera y el alcance y la complejidad de los desempeños establecidos como resultados de aprendizaje de la unidad de trabajo-aprendizaje.

Según Catalano et al. (2004), los diseños curriculares que se organizan por módulos alrededor de una competencia facilitan dicha articulación, no obstante, y en base a la experiencia y apreciación de los autores de este artículo, en las universidades chilenas es más frecuente encontrar diseños mixtos basados en otras organizaciones de las unidades de trabajo-aprendizaje, como ser asignaturas, seminarios o talleres. En estos casos, la clave está en clarificar qué competencia(s) o qué nivel de dominio de una(s) competencia(s) serán abordadas por cada unidad de trabajo-aprendizaje de manera de aportar a su desarrollo. Posteriormente se deberá seleccionar los indicadores de logro que orientarán la planificación didáctica y evaluación. A este fin resulta adecuado el concepto de escalamiento de competencias señalado por 
Vásquez (2010), entendido como la determinación para una misma competencia, de niveles crecientes de desempeño; por ejemplo en función de la complejidad de situaciones que se ofrecen a los estudiantes para estimular su aprendizaje. Para formalizar este desarrollo, Tardif (2003) sugirió la necesidad de prever rigurosamente el escalamiento de las competencias a lo largo de la carrera. Corresponde entonces determinar cuáles competencias serán trabajadas en cada período de la carrera, teniendo en cuenta la dinámica del desarrollo propia de cada competencia y las formas en que éstas se complementan entre sí, tanto en forma horizontal (dentro de un semestre) como vertical (a lo largo de la carrera).

Para realizar lo señalado anteriormente, en las instituciones de educación superior debiera existir una entidad que se haga responsable de la gestión curricular del programa/carrera que sea diferente al responsable de la gestión de la carrera como un todo (por ejemplo directores y/o jefes de carrera), ya que la gestión curricular requiere especial atención y recursos para su realización. Por ejemplo, un comité curricular, podría emprender el desarrollo de los procesos de revisión y actualización curricular con carácter permanente, como parte del compromiso institucional con una cultura por la calidad, y también para hacer frente a las exigencias de los procesos cíclicos de acreditación de carreras. Pero esta tarea de gestión institucional no siempre tiene en cuenta generar niveles de participación colectiva equivalente entre los actores institucionales como los docentes o los estudiantes. Una alternativa que genera posibilidades interesantes de aumentar la participación es el mapeo curricular propuesto por Jacobs (2004), el cual señala un procedimiento que permite generar representaciones visuales del currículum a partir de una matriz con categorías predeterminadas. En la figura 1 se muestra un ejemplo de mapeo curricular. En los procesos de mapeo realizados de manera participativa, los docentes representan gráficamente el currículum real en cada uno de sus cursos y luego revisan en conjunto los mapas para identificar fortalezas, brechas y solapamientos. Una vez completado el proceso, los profesores determinan qué y dónde agregar o eliminar contenidos, o revisan estrategias para generar un plan de carrera mejor integrado. Los mapas curriculares se pueden revisar y actualizar con frecuencia para adaptarlos a necesidades cambiantes de los alumnos 0 del ambiente, y pueden conservarse en sistemas de información para hacerlos más accesibles. El proceso de mapeo curricular responde a tres cuestiones críticas: ¿Quién hace qué?, ¿Cómo se alinea lo que cada uno hace con las metas y estándares establecidos en el perfil de egreso?, ¿Con qué nivel de eficiencia y efectividad estamos trabajando para eso?

\begin{tabular}{|c|c|c|c|c|c|}
\hline \multirow[b]{2}{*}{ Unit Name } & \multicolumn{2}{|c|}{ August } & \multicolumn{2}{|c|}{ September } & \multirow{2}{*}{\begin{tabular}{l}
\multicolumn{1}{c}{ October } \\
Axial Age \\
Qin and Han China \\
Maurya and Gupta \\
India
\end{tabular}} \\
\hline & $\begin{array}{l}\text { Foundations } 8000 B C E- \\
600 B C E \\
\text { Paleolithic and Neolithic } \\
\text { Age } \\
\text { River Valley Civilizations }\end{array}$ & $\begin{array}{l}\text { Foundations } \\
\text { (Continued) } \\
\text { Civilizations' } \\
\text { continued) } \\
\text { Nomadic invasions } \\
\text { Begin Ancient Greece. }\end{array}$ & $\begin{array}{l}\text { Foundations } \\
\text { (Contifued) } \\
\text { Ancient Greece } \\
\text { Roman Republic and } \\
\text { Empire }\end{array}$ & $\begin{array}{l}\text { Foundations } \\
\text { (Continued)-Imperial } \\
\text { Rome Continued } \\
\text { Begin Qin and Han } \\
\text { China }\end{array}$ & \\
\hline $\begin{array}{c}\text { Students will } \\
\text { have an } \\
\text { understanding } \\
\text { of... } \\
\text { (LEARNING } \\
\text { TARGETS) }\end{array}$ & $\begin{array}{l}\text { 1. Locating world } \\
\text { history in the } \\
\text { environment } \\
\text { and time. } \\
\text { 2. Developing } \\
\text { agriculture and } \\
\text { technology } \\
\text { 3. Basic features } \\
\text { of early } \\
\text { civilizations in } \\
\text { different } \\
\text { environments: } \\
\text { culture, state, } \\
\text { social structure }\end{array}$ & $\begin{array}{l}\text { 1. Impact of } \\
\text { interaction } \\
\text { among major } \\
\text { societies }\end{array}$ & $\begin{array}{l}\text { 1. Classical } \\
\text { Civilizations - } \\
\text { understanding major } \\
\text { political } \\
\text { developments, social } \\
\text { and gender structures, } \\
\text { major trading } \\
\text { patterns, and arts, } \\
\text { sciences, and } \\
\text { technology }\end{array}$ & $\begin{array}{l}\text { 1. Major belief } \\
\text { systems - basic } \\
\text { features of major } \\
\text { world belief systems } \\
\text { prior ro } 600 \mathrm{CE} \text { and } \\
\text { where each belief } \\
\text { system applied by } 600 \\
\text { CE }\end{array}$ & $\begin{array}{l}\text { Major Belief Systems } \\
\text { (Continued) - } \\
\text { polytheism, Judaism, } \\
\text { Christianity, } \\
\text { Confucianism, } \\
\text { Daoism, Legatism, } \\
\text { Hinduism, and } \\
\text { Buddhism }\end{array}$ \\
\hline $\begin{array}{l}\text { Essential } \\
\text { Questions }\end{array}$ & $\begin{array}{l}\text { What is world history? } \\
\text { What has been the } \\
\text { biggest cultural change } \\
\text { in world history? } \\
\text { How did location impact } \\
\text { the development of } \\
\text { early societies? }\end{array}$ & $\begin{array}{l}\text { Why are people willing } \\
\text { to give up a nomadic } \\
\text { lifestyle for a settled } \\
\text { existence? } \\
\text { How did nomadic } \\
\text { invasions encourage } \\
\text { the development of } \\
\text { new technology? }\end{array}$ & $\begin{array}{l}\text { Was ancient Greece a } \\
\text { true democracy? } \\
\text { Does art, architecture, } \\
\text { and literature reflect } \\
\text { the true values of a } \\
\text { society? }\end{array}$ & $\begin{array}{l}\text { How does religion } \\
\text { create unity and } \\
\text { conflict? } \\
\text { Can rules be too } \\
\text { strict? }\end{array}$ & $\begin{array}{l}\text { Why dont } \\
\text { empires last? } \\
\text { (Continued) } \\
\text { How do refigions } \\
\text { or philosophies } \\
\text { influence political } \\
\text { rule? }\end{array}$ \\
\hline $\begin{array}{l}\text { Assessment } \\
\text { Strategies } \\
\text { Formative \& } \\
\text { Summative }\end{array}$ & $\begin{array}{l}\text { Human Drama Book- } \\
\text { Paleolithic vs. Neolithic } \\
\text { Human Origins Collage } \\
\text { activity } \\
\text { DBQ outine } \\
\text { Comparative Essay }\end{array}$ & $\begin{array}{l}\text { Unit Test } \\
\text { Assess Living History } \\
\text { Park project }\end{array}$ & $\begin{array}{l}\text { Athens/ Sparta Debate } \\
\text { Ancient Greece DBQ } \\
\text { essay } \\
\text { Greece Test }\end{array}$ & $\begin{array}{l}\text { Chinese Emperor/ } \\
\text { Empress Story } \\
\text { Comparison Travel } \\
\text { posters - Rome and } \\
\text { Han China } \\
\text { Multiple Choice } \\
\text { Quizzes } \\
\text { Comparison Essay }\end{array}$ & $\begin{array}{l}\text { Role-Plays - caste } \\
\text { system } \\
\text { Multiple Choice Test } \\
\text { Comparison Essay } \\
\text { DBQ essay }\end{array}$ \\
\hline
\end{tabular}

Fig. 1: Ejemplo de mapeo curricular.

La cultura académica tradicional ha tendido a considerar "propietarios" de los cursos a individuos o unidades académicas, más que a la carrera como unidad de sentido. El efecto es un currículum formado por asignaturas yuxtapuestas, con baja coherencia y poca sinergia. El proceso de mapeo puede identificar "recorridos curriculares" o secuencias de cursos relacionados en términos de contenido y que tienen 
responsabilidades compartidas en la generación de conocimiento y competencia de los alumnos. En Britton et al. (2008), la tarea de mapeo curricular la realizan los docentes en grupo de pares, para facilitar la apropiación del proceso, y los resultados se acumulan en un sistema de información ad hoc, para facilitar la visualización.

Los mapas curriculares sirven como herramientas para evaluar la consistencia de una carrera en su conjunto, dando una vista general de la estructura y señalando la contribución de las asignaturas individuales a las metas de la carrera. Los mapas curriculares también pueden servir para identificar fortalezas y debilidades, por ejemplo al detectar en ellos competencias que se atienden en pocos cursos, revisar si la secuencia de los cursos es la óptima, entre otras. Se entiende que los datos surgidos del mapeo curricular pueden servir para generar compromiso con el cambio, teniendo en cuenta que para que el cambio sea exitoso, se debe dar una combinación adecuada de demanda y apoyo a todos los involucrados (Fullan, 2003).

En el contexto de las carreras de Ingeniería, otra propuesta para la organización de currículo ha tomado fuerza, la cual se denomina CDIO. La iniciativa CDIO es propuesta por Edward et al. (2007) y ha sido utilizada como marco de referencia para apoyar los procesos de rediseño curricular en carreras de ingeniería. Esta iniciativa identifica las diferentes perspectivas de la formación del Ingeniero: su dimensión disciplinar, su dimensión personal y profesional, sus habilidades interpersonales y su capacidad para concebir, diseñar, implementar y operar (de ahí su sigla CDIO) soluciones a los problemas de su entorno. Por lo tanto, para alinear un currículo de ingeniería con esta iniciativa, un paso necesario es verificar si las competencias están identificadas, si han sido establecidos los bloques básicos de formación para la carrera y las asignaturas que lo constituyen, y si están claramente establecidas las responsabilidades por el desarrollo de las competencias identificadas en cada una de las asignaturas. Cuando no haya evidencia de dicha asignación de responsabilidades, será necesario definir un proceso que permita asignar responsabilidades por el desarrollo de las competencias a los bloques de formación y a las asignaturas que los componen. Ello implica efectuar una revisión a nivel de micro currículo de las asignaturas, para integrar explícita y efectivamente el desarrollo de la competencia.

Según Schmal y Ruiz-Tagle (2008), en la literatura no existe un método formal que permita construir un currículo basado en competencias, pero si hay diversas experiencias que señalan las actividades realizadas, recursos utilizados y marco regulatorio que los rige. Las experiencias consideradas en este articulo (Schmal y Ruiz-Tagle, 2008), (Vásquez et al, 2011), (Goñi y Meseguer, 2010) si bien poseen aspectos comunes, poseen variaciones que pueden ser ocasionadas por características propias de la disciplina (carrera).

Los referentes teóricos discutidos en esta sección permitieron definir una metodología de trabajo para revisar y actualizar el rediseño curricular realizado en la carrera de Ingeniería Civil en Computación e Informática de la Universidad Arturo Prat. Específicamente, el escalamiento de las competencias propuestas por Tardif (2003), la generación de representaciones visuales del currículum propuestas por Jacobs (2004) y la identificación de las diferentes perspectivas de la formación de un Ingeniero, propuestas por Edward et al. (2007) en CDIO, son las bases de la metodología de trabajo propuesta.

\section{METODOLOGÍA DE TRABAJO}

Al finalizar el proceso de rediseño curricular de la carrera de Ingeniería Civil en Computación e Informática en el año 2012, la dirección de la carrera decidió evaluar los productos obtenidos de éste proceso, como forma de asegurar la calidad del currículo generado. Para lo anterior se definió una metodología que considera los artefactos producidos en el proceso de rediseño, con el objeto principal de establecer una coherencia entre los artefactos ya generados y un desarrollo equilibrado de las competencias a lo largo del proceso de aprendizaje. Esta metodología consiste en 5 etapas: procesamiento pedagógico de las competencias establecidas en el perfil, elaboración de una secuencia de progresión de las competencias, análisis sobre el aporte de las asignaturas al desarrollo de las competencias, actualización de los programas y de la malla curricular y, producción de pruebas de logros.

Las etapas anteriormente señaladas se realizan en secuencia, tal como lo muestra la Figura 2, permitiéndose la retroalimentación (y posterior revisión de las etapas ya realizadas), con el objeto de actualizar y mantener la coherencia y consistencia entre los artefactos. Para aplicar la metodología, esta requiere de los artefactos producidos durante el rediseño curricular: competencias del egresado, programas de asignaturas, malla y pruebas de logros. Estos artefactos pueden ser utilizados en una o más etapas. Por otra parte, los artefactos que la metodología debe generar son: malla y programas de asignaturas actualizados, pruebas de logros actualizadas y mapa de progresión en el aprendizaje. 


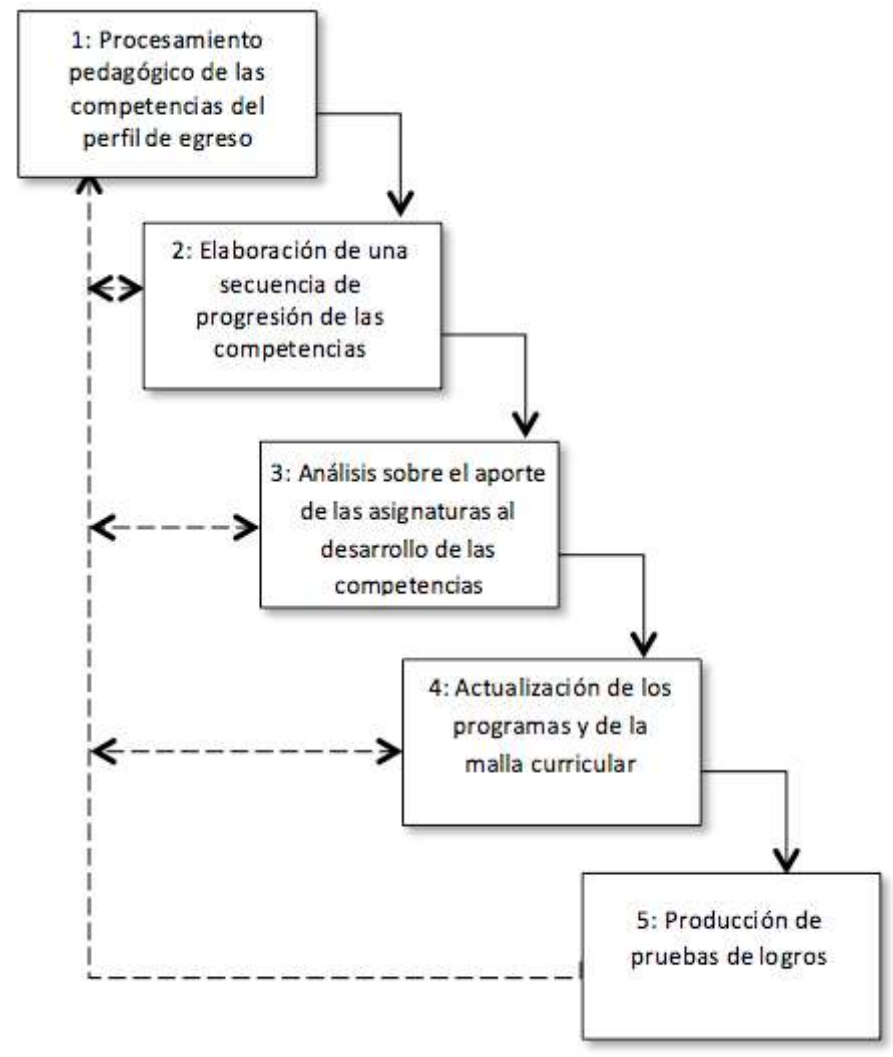

Fig. 2: Metodología para la revisión y actualización de un rediseño de currículo basado en competencias.

En la Figura 3, a través de un diagrama de flujo de trabajo, se muestran las diferentes etapas de la metodología (rectángulos) y los artefactos que requieren (flechas que ingresan a los rectángulos), así como también los artefactos que generan (flechas que salen de los rectángulos) y son utilizados por las otras etapas de metodología o como resultado del proceso.

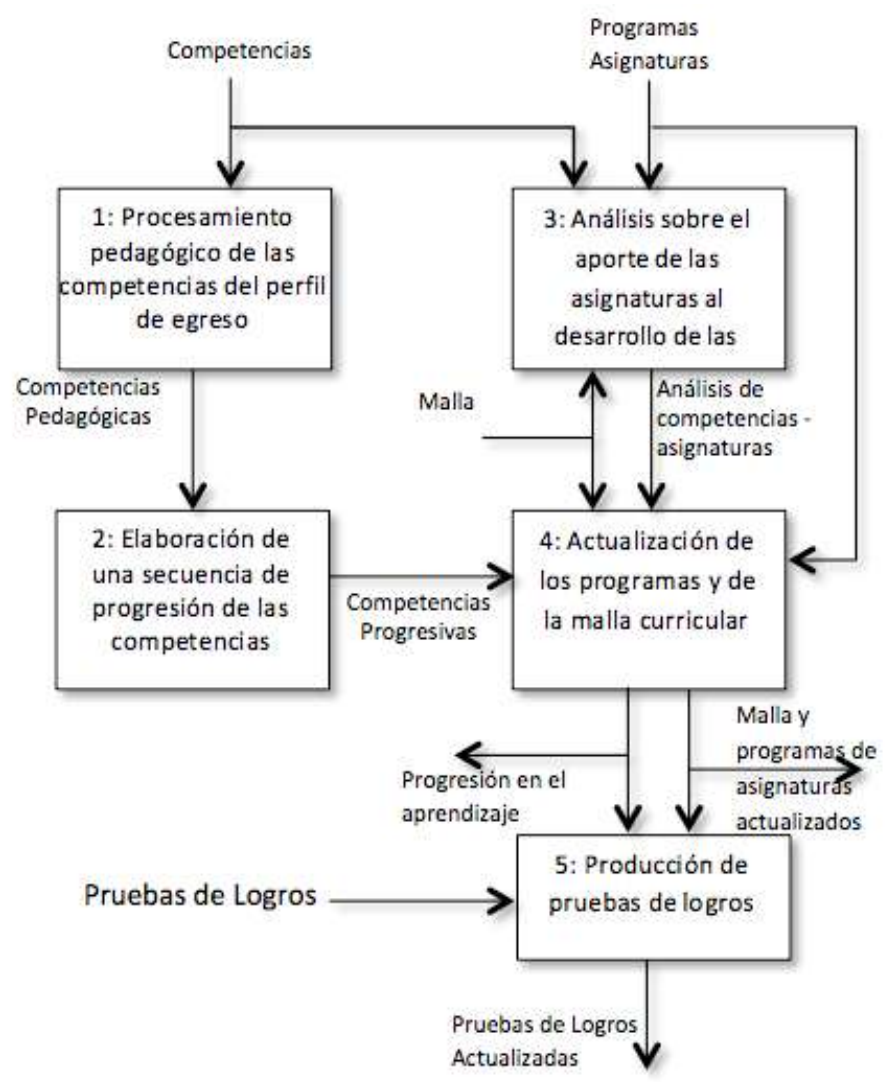

Fig. 3: Diagrama de flujo de trabajo para la metodología. 


\section{Etapa 1: Procesamiento pedagógico de las competencias establecidas en el perfil}

El objetivo de esta etapa es generar versiones pedagógicas de las competencias a partir del análisis de las competencias que forman el perfil de egreso de la carrera. Debido a que las competencias del perfil de egreso fueron formuladas en acuerdo con la comunidad de usuarios y profesionales, estas describen el quehacer competente de un graduado, a diferencia de las versiones pedagógicas de las competencias que describen el quehacer de un estudiante antes de graduarse. Estas versiones pedagógicas de las competencias permiten generar elementos orientadores concretos para seleccionar asignaturas, cursos 0 módulos (la estructuración de la malla curricular o aspecto macrocurricular), y para dirigir la organización de la enseñanza y la evaluación (aspecto microcurricular). Para el desarrollo de esta etapa, se requieren de las competencias definidas en el perfil de egreso para la formulación de una versión de éstas en un contexto pedagógico. Luego, estas versiones son analizadas y validadas a través de reuniones sucesivas con los docentes de la carreras, ya que ellos son los responsables de los procesos de enseñanza-aprendizaje. El resultado de esta etapa es un documento que contiene las versiones pedagógicas de cada una de las competencias del perfil de egreso.

\section{Etapa 2: Elaboración de una secuencia de progresión de las competencias.}

El objetivo de esta etapa es desagregar las competencias del perfil en niveles o escalones. Para el desarrollo de esta etapa, se requieren de las versiones pedagógicas de cada una de las competencias del perfil de egreso, obtenidas en la etapa 1. Posteriormente, los docentes de la carrera deben definir en cuantos niveles desagregarán las competencias. Luego, para cada competencia, y en cada nivel, corresponde acordar el tipo de evidencia que deben producir los alumnos para que se reconozca como alcanzado. Lo anterior puede realizarse a través de sucesivas reuniones acordando y utilizando una representación gráfica que permita verificar si las evidencias propuestas reflejan una progresión en el desarrollo de la competencia. El resultado generado en esta etapa es un artefacto, por ejemplo una matriz, tal como lo muestra la figura 4, que señale las evidencias (o productos) que el estudiante debe ser capaz de realizar, para cada nivel de cada competencia. Insumos para esta etapa son la versión pedagógica de las competencias y su resultado es un mapa progresivo de evidencias de cada competencia.

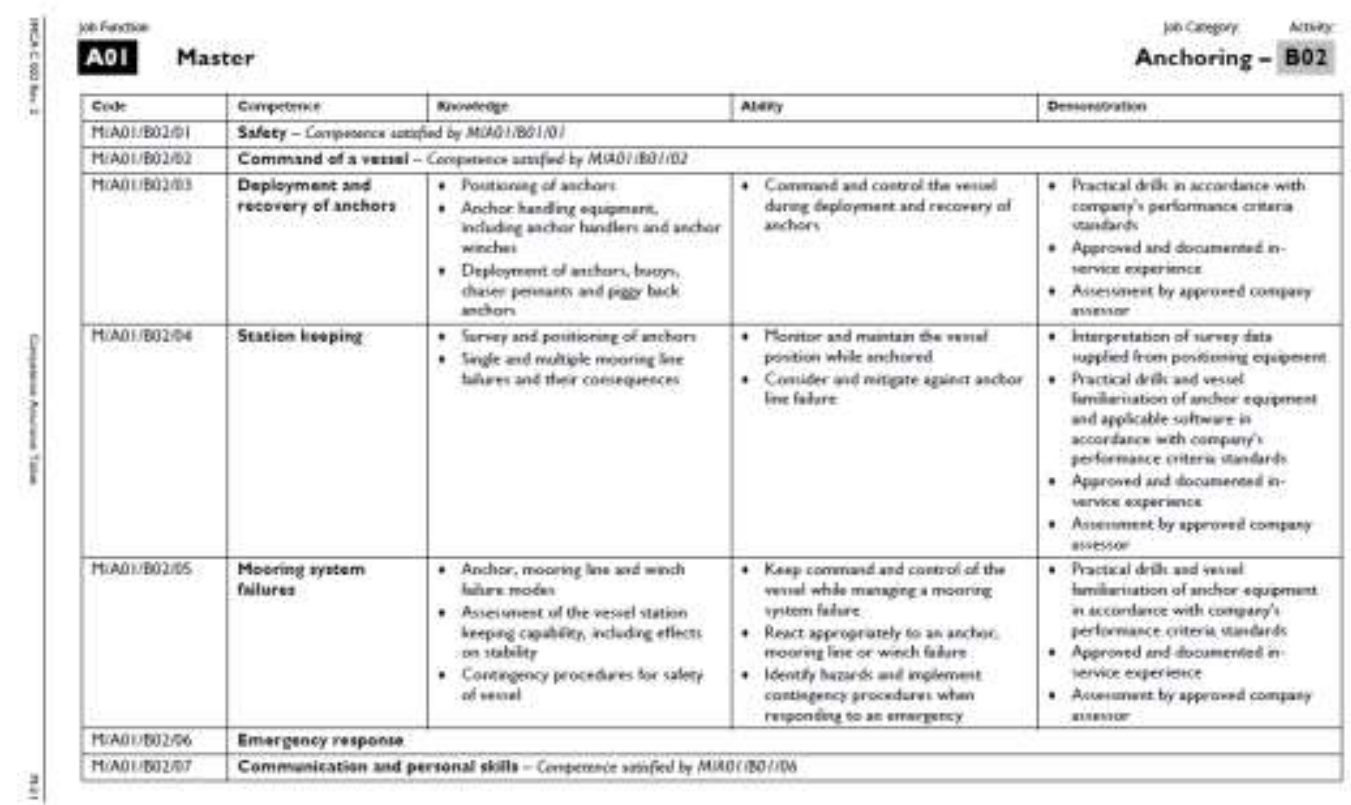

Fig. 4: Ejemplo de detalle de evidencias para una competencia

Etapa 3: Análisis sobre el aporte de las asignaturas al desarrollo de las competencias.

El objetivo de esta etapa es analizar la existencia de la progresión del aprendizaje y desarrollo de las competencias según lo establecido en el conjunto de programas de la malla curricular. Para el desarrollo de esta etapa, se requieren de las competencias del perfil de egreso, los programas de asignaturas y la malla curricular, artefactos que son resultado del proceso de rediseño curricular. Con estos elementos, un profesor debe revisar las competencias señaladas en cada unidad de trabajo-aprendizaje y establecer cuales y cuantas competencias son abordadas en cada nivel de la malla curricular. Para realizar esta tarea es conveniente utilizar una herramienta gráfica, que permita evidenciar la mayor o menor concentración de competencias señaladas en los programas de asignaturas en los distintos niveles de la malla. 
A partir del análisis se registra: (i) si hay alguna(s) competencia(s) sobre la(s) que se trabaja relativamente poco en el conjunto de materias (competencias poco atendidas); (ii) si hay alguna(s) competencia(s) que aparece(n) mencionada(s) muchas veces a lo largo de la carrera (competencias sobrerrepresentadas); y (iii) si el despliegue temporal de las competencias se distribuye de manera pareja a lo largo de toda la carrera 0 si se concentra el desarrollo de determinada(s) competencia(s) en un período de tiempo muy corto (p.ej. los últimos semestres de la carrera).

El resultado generado en esta etapa debe ser un artefacto que muestre el desarrollo de las competencias definido en el conjunto de programas, el cual puede ser representado en formato de matrices u otras herramientas para este fin, y las respectivas conclusiones al analizar el artefacto.

\section{Etapa 4: Actualización de los programas y de la malla curricular.}

El objetivo de esta etapa es actualizar los programas de asignaturas y la malla curricular. En esta etapa, se producen las recomendaciones para aumentar la correlación entre la malla, los programas y el perfil de egreso. Esto implica atender las conclusiones obtenidas en la etapa anterior y, por ejemplo, seleccionar qué competencias poco atendidas hay que abordar más tempranamente en las asignaturas de la malla, qué competencias sobrerrepresentadas hay que eliminar de algunos programas de asignatura, y revisar que el desarrollo de las competencias a lo largo de la malla siga una progresión adecuada. Para el desarrollo de esta etapa se requiere de los resultados del análisis realizado en la etapa 3 y los niveles con las evidencias de las competencias de la etapa 2, además de los programas de asignaturas y la malla curricular, obtenidos del proceso de rediseño. En base a estos artefactos, los profesores deben reunirse para acordar y modificar, si fuese necesario, los programas de asignaturas al menos en aspectos relacionados a: competencias y su nivel de desarrollo, resultados de aprendizaje y métodos de evaluación con el objeto de obtener un programa consistente y coherente. Lo anterior deberá realizarse considerando una progresión sostenida y equilibrada en el desarrollo de las competencias. El resultado de esta etapa son programas de asignaturas actualizados, una malla actualizada y un mapa de la progresión del desarrollo para cada una de las competencias en cada nivel de la malla. Este mapa puede ser implementado a través de gráficos.

\section{Etapa 5: Producción de pruebas de Logros.}

El objetivo de esta etapa es establecer y definir los mecanismos que permitirán medir el desarrollo de las competencias en los estudiantes. Para desarrollar esta etapa se requiere de los artefactos producidos en la etapa 4: programas de asignaturas actualizados, malla curricular actualizada y el mapa de la progresión del desarrollo de las competencias. Una vez que los programas han sido actualizados, los profesores deben reunirse para acordar un determinado momento de la carrera, por ejemplo hacia la mitad y hacia el final, y definir a partir de las evidencias definidas en las competencias y sus niveles para ese momento, según lo especificado en el mapa de la progresión del desarrollo de las competencias, un conjunto de situaciones integradoras de evaluación que permitan medir si los estudiantes están llegando al logro esperado. El resultado de esta etapa será la definición de una o varias instancias que permitan evaluar el grado de desarrollo de las competencias en el estudiante (prueba de logros). Estas instancias pueden ser pruebas escritas, portafolios con artefactos específicos, informes externos, prácticas profesionales, entre otras.

\section{CASO DE ESTUDIO: INGENIERÍA CIVIL EN COMPUTACIÓN E INFORMÁTICA}

La carrera de Ingeniería civil en Computación e Informática, es una carrera impartida por la Universidad Arturo Prat, la cual pertenece al Estado de Chile. La carrera inicia sus actividades el año 2000 con un plan curricular de 60 asignaturas y 6 años de duración. Luego el 2007 realiza un primer rediseño curricular estableciendo un nuevo plan curricular con 55 asignaturas y 5,5 años de duración. Ambos planes estaban orientados por objetivos. Durante el 2011 la carrera se somete a un proceso de acreditación de forma voluntaria, obteniendo 4 años de acreditación. Debido a que el proceso de acreditación de la carrera detectó algunas debilidades curriculares y sumado a la intención de la Universidad de contar con carreras basadas en competencias, la dirección de la carrera definió la necesidad de realizar un nuevo rediseño curricular. Este rediseño fue realizado durante el 2012 con el objeto de actualizar su currículo y cumplir los acuerdos del proceso de acreditación de carreras realizado el 2011. De este rediseño se obtuvo un perfil de egreso basado en 11 competencias (4 competencias genéricas establecidas por la Universidad para todas las carreras que imparte, 2 competencias establecidas por la Facultad para todas las carreras de ingeniería y 5 competencias específicas de la disciplina de computación e informática), un plan curricular a 5 años (10 semestres) y nuevos y actualizados programas de asignaturas como los principales cambios al plan anterior. Durante este proceso, los profesores de la carrera generaron programas de asignaturas proponiendo que competencias la asignatura aportaría en su desarrollo, los resultados de aprendizajes (lo que se espera que el estudiante aprenda y apoyen al desarrollo de las competencias), los contenidos, las formas de evaluación y los recursos para la enseñanza. Este proceso de rediseño fue liderado por el Director de Carrera y con el apoyo del cuerpo docente de la carrera, constituido por 10 profesores. 
Actualmente, el nuevo plan curricular tiene tres admisiones (2013, 2014 y 2015), por lo que no existen resultados preliminares sobre el impacto en los estudiantes, ya que las pruebas de logros (definidas al ejecutar esta metodología) aún no han sido aplicadas. Con la intención de revisar las propuestas de los profesores en función de un aprendizaje progresivo, el cual pudiera ser medido a través de pruebas de logros, se aplicó la propuesta metodológica definida anteriormente.

\section{Etapa 1: Procesamiento pedagógico de las competencias establecidas en el perfil.}

En esta etapa se analizaron las 11 competencias que considera el perfil de egreso para la carrera y se reformularon considerando el proceso de aprendizaje del estudiante. Por ejemplo, dada la competencia "Diseña, construye e integra soluciones tecnológicas considerando elementos de software, hardware, telecomunicaciones y automatización, para agregar valor a las organizaciones públicas y privadas, y a través de éstas a la sociedad", resulta obvio que no se puede evaluar el avance del estudiante en esta competencia a través de una prueba de logros, ya que su puesta en juego requiere del ambiente externo y del juicio de la organización respecto de si el sujeto agregó o no valor con su propuesta. Para evaluar el desarrollo de la competencia durante el aprendizaje del estudiante, esta fue reformulada como sigue: "EI egresado ha dado muestras de que puede diseñar y construir soluciones tecnológicas para organizaciones (pueden ser simulaciones) que integran elementos de software, hardware, telecomunicaciones y automatización." Esta nueva versión de la competencia sí permitiría diseñar una prueba de logro: bastaría con proponer al estudiante una simulación de una situación real para que él trate de producir una solución utilizando sus conocimientos y de esta manera evaluar el desarrollo de la competencia.

\section{Etapa 2: Elaboración de una secuencia de progresión de las competencias.}

Para que un estudiante pueda desarrollar las competencias de forma progresiva, éstas deben estar explícitamente señaladas en el programa de asignatura. Para materializarlo en el caso de estudio, fue necesario realizar dos tareas: definir niveles de avance y evidencias asociadas a dichos niveles. Para determinar la cantidad de niveles, primero se definieron los productos que un estudiante debería realizar para considerar que ha alcanzado el desarrollo de la competencia. En función de la cantidad de productos definidos, se acordó que la cantidad de niveles adecuada era 3 y se denominaron inicial, intermedio y avanzado. Para la definición de los productos y niveles de cada competencia, fue necesario un trabajo conjunto donde el grupo de profesores de la carrera lograran un acuerdo. En la Tabla 1 se observan los niveles y los productos asociados a cada nivel para una de las competencias de la carrera.

Tabla 1. Niveles y evidencias por nivel para la competencia de Diseño de soluciones informáticas.

\begin{tabular}{|c|c|c|c|}
\hline \multirow[t]{2}{*}{ Competencia } & \multicolumn{3}{|c|}{$\begin{array}{l}\qquad \text { Detalle por nivel } \\
\text { trena en clase y en la evaluación se le demanda evidencias de producto para que: }\end{array}$} \\
\hline & Inicial & Intermedio & Avanzado \\
\hline $\begin{array}{l}\text { Soluciones informáticas } \\
\text { (DISSOL) } \\
\text { Diseña, construye e } \\
\text { integra soluciones } \\
\text { tecnológicas considerando } \\
\text { elementos de software, } \\
\text { hardware, telecomuni- } \\
\text { caciones y automatiza- } \\
\text { ción, para agregar valor a } \\
\text { las organizaciones } \\
\text { públicas y privadas, y a } \\
\text { través de éstas a la } \\
\text { sociedad. }\end{array}$ & $\begin{array}{l}\text { Construya algoritmos } \\
\text { utilizando un lenguaje de } \\
\text { programación } \\
\text { Implemente medios de } \\
\text { comunicación entre } \\
\text { dispositivos digitales. } \\
\text { Realice un levantamiento de } \\
\text { requerimientos }\end{array}$ & $\begin{array}{l}\text { Implemente una solución } \\
\text { informática con metodologías } \\
\text { orientadas a objetos o } \\
\text { estructurados a partir de un } \\
\text { diseño. } \\
\text { Diseñar un sistema de comu- } \\
\text { nicaciones utilizando protoco- } \\
\text { los y hardware apropiado }\end{array}$ & $\begin{array}{l}\text { Realice un diseño e } \\
\text { implementación de } \\
\text { sistemas de software } \\
\text { orientados a objetos o } \\
\text { estructurado, utilizando un } \\
\text { proceso de desarrollo de } \\
\text { software. } \\
\text { Realice un diseño e } \\
\text { implementación de } \\
\text { sistemas de software de } \\
\text { apoyo a la toma de } \\
\text { decisiones. }\end{array}$ \\
\hline
\end{tabular}

Etapa 3: Análisis sobre el desarrollo de las competencias en las asignaturas.

En la realización de esta etapa se analizaron los programas de asignaturas con el objeto de determinar la relación entre asignaturas y competencias. Para lo anterior se traspasó a una matriz la información de las competencias consideradas según lo señalado por el programa de asignatura. En la matriz, las filas corresponden a las asignaturas y las columnas a las competencias. En la Tabla 2 se muestra un extracto correspondiente a las competencias genéricas y específicas del 1er y 2do semestre de la malla. 
Tabla 2. Competencias por asignatura, ejemplo para el primer y segundo semestre.

\begin{tabular}{|c|c|c|c|c|c|c|c|c|c|c|c|c|}
\hline & & \multicolumn{4}{|c|}{ Genéricas } & \multicolumn{7}{|c|}{ Específicas } \\
\hline & & হి & 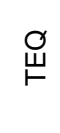 & 气 & $\begin{array}{l}\text { O } \\
\text { O } \\
\text { D) }\end{array}$ & $\begin{array}{l}\frac{0}{\frac{\pi}{\alpha}} \\
\frac{\tilde{\alpha}}{\tilde{r}}\end{array}$ & $\begin{array}{l}\text { E } \\
0 \\
0 \\
0\end{array}$ & $\begin{array}{l}\vec{D} \\
\mathbb{D} \\
\underline{C D} \\
0\end{array}$ & $\sum_{\underline{1}}^{0}$ & $\begin{array}{l}\grave{o} \\
\frac{\pi}{a}\end{array}$ & 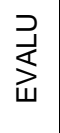 & $\underset{\mathrm{N}}{\underline{0}}$ \\
\hline 1 & Cálculo diferencial & & & & $x$ & $x$ & & & & & & \\
\hline & Álgebra & & & & $\mathrm{x}$ & $\mathrm{x}$ & & & & & & \\
\hline & Fundamentos de Química & & & & $\mathrm{x}$ & $\mathrm{x}$ & & & & & & \\
\hline & Introducción a la ingeniería & & $x$ & & & $\mathrm{x}$ & & & & & & \\
\hline & Electivo formación general 1 & $\mathrm{x}$ & $\mathrm{x}$ & & & & & & & & & \\
\hline 2 & Cálculo Integral & & & & $x$ & $x$ & & & & & & \\
\hline & Álgebra Lineal & & & & $\mathrm{x}$ & $\mathrm{x}$ & & & & & & \\
\hline & $\begin{array}{ll}\begin{array}{l}\text { Entrenamiento } \\
\text { personales }\end{array} & \text { Habilidades } \\
\end{array}$ & & $\mathrm{x}$ & & & & $\mathrm{x}$ & & & & & \\
\hline & $\begin{array}{l}\text { Introducción a la programación } \\
\text { computacional }\end{array}$ & & & & $\mathrm{x}$ & $x$ & & & & & & \\
\hline & Electivo formación general 2 & $\mathrm{x}$ & $x$ & & & & & & & & & \\
\hline
\end{tabular}

Al analizar la información, correspondiente a los 10 semestres que dura el plan curricular, se observó que en el primer año de la carrera (semestre 1 y 2), muchas materias eligen como única competencia específica para apoyar en su desarrollo a la competencia de formulación y resolución de problemas (FRPRO), y entre las genéricas, hay un alto énfasis en la referida al uso de las tecnologías de la información y comunicación (USOTIC). Por otra parte, la competencia sobre evaluación y uso de estándares de calidad en la profesión (EVALU) aparecía considerada en una sola asignatura de la malla. Esto da cuenta de un desequilibrio entre las competencias a desarrollar y la cantidad de asignaturas que asumen acciones de enseñanza para apoyar su desarrollo. Para visualizar mejor el análisis, se desarrolló una matriz donde se muestra la frecuencia en que la competencia es abordada en los programas de asignatura, a lo largo de los 10 semestres de la carrera. La Tabla 3 muestra y confirma claramente que hay competencias consideradas en varias asignaturas y otras que son consideradas en pocas, incluso una de las competencias es considerada una sola vez.

La principal conclusión obtenida en esta etapa es que las competencias de la carrera no estaban distribuidas entre las asignaturas de forma adecuada, teniendo en cuenta que: (a) No se evidencia una progresión en el desarrollo de las competencias; (b) No resulta razonable pensar que una competencia pueda ser desarrollada completamente por solo una asignatura; y (c) Es posible desarrollar competencias en menos tiempo que el propuesto en la situación actual.

Tabla 3. Cantidad de asignaturas que consideran la competencia en un nivel de la malla.

\begin{tabular}{|c|c|c|c|c|c|c|c|c|c|c|c|}
\hline 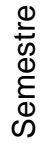 & $\sum_{0}^{2}$ & $\begin{array}{l}\text { O } \\
\end{array}$ & 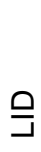 & $\begin{array}{l}\frac{0}{5} \\
0 \\
\mathcal{O}\end{array}$ & $\begin{array}{l}0 \\
0 \\
\frac{\pi}{0} \\
\frac{\pi}{\tilde{r}} \\
\frac{\pi}{u}\end{array}$ & $\begin{array}{l}\text { Ł } \\
\text { Ò } \\
\text { ○ }\end{array}$ & $\begin{array}{l}\vec{D} \\
\mathscr{D} \\
\frac{\mathscr{D}}{\square}\end{array}$ & $\sum_{\underline{z}}^{n}$ & 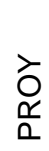 & 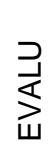 & 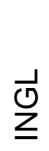 \\
\hline 1 & 1 & 2 & & 3 & 4 & & & & & & \\
\hline 2 & 1 & 2 & & 3 & 3 & 1 & & & & & \\
\hline 3 & 2 & 1 & 1 & 3 & 4 & 1 & 1 & & & 1 & \\
\hline 4 & 3 & 2 & & 3 & 5 & & 1 & & & & \\
\hline 5 & 2 & 2 & & 3 & 3 & 2 & 2 & & 1 & & \\
\hline 6 & & 3 & 1 & 2 & & 3 & 3 & & 1 & & 1 \\
\hline 7 & 1 & 3 & & & 1 & 2 & & 2 & & & \\
\hline 8 & 2 & 1 & 2 & 3 & 3 & 4 & 3 & 1 & 3 & & \\
\hline 9 & 2 & 2 & 1 & 4 & 3 & 4 & 4 & 1 & 2 & & 1 \\
\hline 10 & 3 & & & 4 & 2 & 3 & 2 & & & & 1 \\
\hline
\end{tabular}


Con los resultados del análisis anterior y los escalones de competencias, fue preciso utilizar la malla como elemento de planificación mostrando de manera sintética el proceso de avance a lo largo de las asignaturas, de modo que se generó un instrumento de comunicación con las diferentes asignaturas y que permitió revisar sus programas y ajustar sus metodologías de manera acorde. En la Tabla 4 se muestra el ajuste propuesto para el tercer semestre de la malla curricular (2do año), el cual señala los niveles de desarrollo de la competencia que las asignaturas deben abordar para una o más competencias. Esta tabla es realizada para todos los semestres de la malla curricular.

Tabla 4. Grado de desarrollo de la competencia por asignatura para el semestre 3: Int=Intermedio; Ini=Inicial

\begin{tabular}{|c|c|c|c|c|c|c|c|c|c|c|c|}
\hline \multirow{2}{*}{ Asig. } & \multicolumn{3}{|c|}{ Genéricas } & \multicolumn{8}{|c|}{ Específicas } \\
\hline & COMU & TEQ & LID & USOTIC & FRPROB & DPCONT & DISSOL & INVAP & PROY & EVALU & INGL \\
\hline $\begin{array}{l}\text { Calculo } \\
\text { Vectorial }\end{array}$ & & & & Int & Int & & & & & & \\
\hline $\begin{array}{l}\text { Ecuaciones } \\
\text { diferenciales }\end{array}$ & & & & Int & Int & & & & & & \\
\hline $\begin{array}{l}\text { Cinemática y } \\
\text { Dinámica }\end{array}$ & Ini & Ini & & & Int & & & & & & \\
\hline $\begin{array}{l}\text { Programación } \\
\text { Avanzada }\end{array}$ & & & & & Int & Ini & & Ini & & & \\
\hline $\begin{array}{l}\text { Organización } \\
\text { de Empresas }\end{array}$ & Int & & & Int & & & & & & Ini & \\
\hline
\end{tabular}

Con la información de la Tabla 4 (para todos los semestres del plan curricular) se genera un gráfico de burbujas para cada competencia, con lo cual puede observarse el desarrollo de esta. El eje horizontal del gráfico corresponde a los semestres de la malla curricular, y el eje vertical a los niveles de desarrollo de la competencia representados por los números 1 (inicial), 2 (intermedio) y 3 (avanzado). En la intersección semestre-nivel de desarrollo, se puede apreciar a través del tamaño de una burbuja, si el nivel de desarrollo para la competencia en ese semestre, está siendo abordada o no y en qué cantidad. Estos gráficos tendrán "buena forma" si se observa que la competencia se va desarrollando a lo largo de toda la carrera con niveles de progresión crecientes, y que no hay excesiva concentración de asignaturas abordando el nivel de desarrollo de la competencia en determinados semestres (tamaño de la burbuja). La Figura 5 muestra un ejemplo para la competencia de comunicación (COMU), en el cual se aprecia que: (a) La competencia posee un desarrollo progresivo; (b) En el primer semestre hay más asignaturas que abordan el nivel de desarrollo inicial, a diferencia de los otros semestres; y (c) En el séptimo semestre no hay asignaturas que abordan la competencia.

Comunica ideas, argumentos, conocimientos de manera clara y eficaz, tanto de forma oral como escrita, utilizando los medios adecuadamente y adaptándose a las características de la situación y de la audiencia

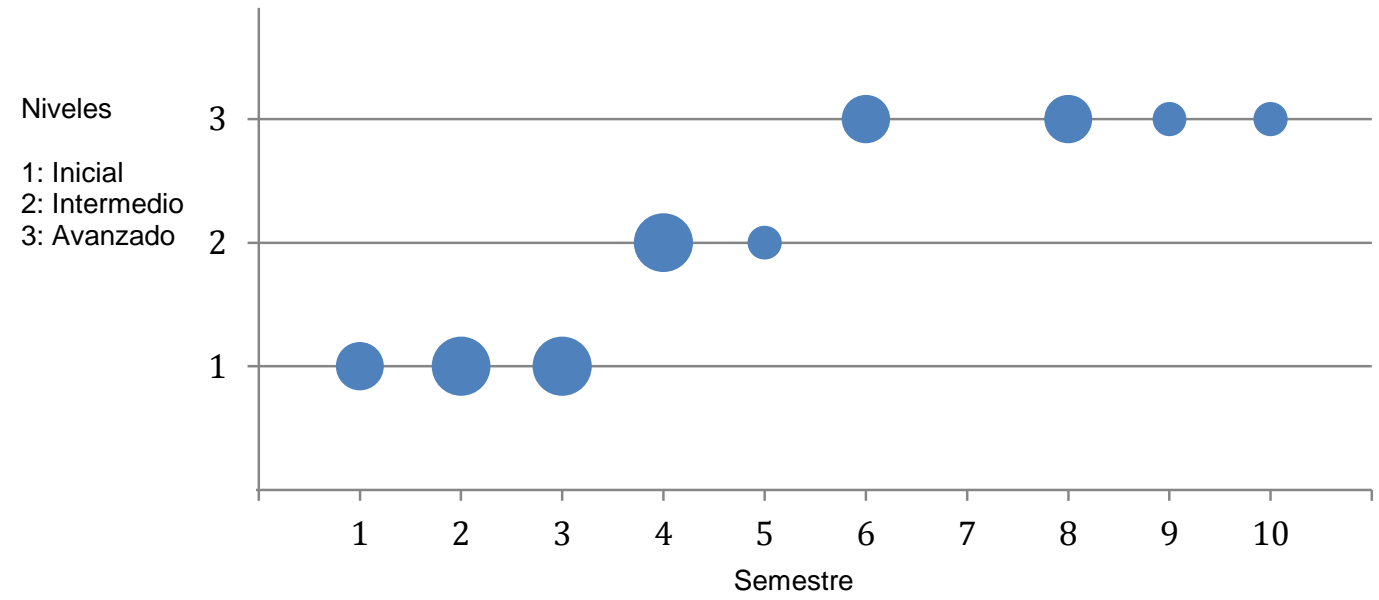

Fig 5: Progresión en el desarrollo de la competencia y concentración de asignaturas que la abordan. 


\section{Etapa 4: Actualización de los programas y de la malla curricular.}

Una vez que cada asignatura tiene clara su contribución al desarrollo de las competencias y el nivel de desarrollo con que aporta, la dirección de carrera solicita a los profesores modificar y actualizar los programas de asignaturas, no solo en términos de eliminar y/o agregar competencias sino que además establecer resultados de aprendizaje, metodologías de aprendizaje y métodos de evaluación acordes a los niveles de desarrollo de la competencia en que debe ser abordado por la asignatura.

\section{Etapa 5: Producción de pruebas de Logros.}

Para la confección de la prueba de logros, se utilizaron los gráficos generados en la etapa 3 , ya que estos pueden mostrar el nivel de desarrollo alcanzado en las competencias, en un semestre de la malla curricular. En el caso de la carrera, la malla establece dos prácticas profesionales, una en el nivel 5 y otra en el nivel 7 y se determinó que estas actividades, y el trabajo final para la obtención del título profesional, operarían como instrumentos para medir el logro de las competencias en el estudiante.

Con respecto al uso de las prácticas profesionales como pruebas de logros, la razón de esta decisión es que estas prácticas son realizadas en entidades externas a la carrera, y evaluadas por ellas, lo que hace la evaluación más objetiva. La evaluación de las prácticas se realiza a través de una evaluación confidencial y un informe escrito. La evaluación confidencial debe ser contestada por el jefe directo del estudiante mientras realizó su práctica y el informe escrito debe ser realizado por el estudiante y evaluado por un profesor de la carrera. Como las prácticas son realizadas en entidades externas, no es seguro que las labores a realizar por los estudiantes permitan evaluar el logro de todos los niveles de desarrollo de las competencias para esa práctica. Por esta razón los estudiantes deben realizar prácticas profesionales en organizaciones que requieran labores que permitan evaluar la mayor cantidad de competencias. Para lo anterior, el director de carrera debe orientar a los estudiantes en la búsqueda y selección del lugar en donde realizará la práctica profesional. En el caso que la evaluación confidencial y/o la evaluación del informe escrito demuestren que el estudiante no ha logrado desarrollar alguna(s) competencias, se deberá, primero, definir actividades remediales que ayuden al estudiante lograr el desarrollo de la(s) competencia(s). Las actividades serán propuestas por el mismo profesor que estuvo a cargo de la evaluación de la práctica. Además, y en caso de ser necesario, se puede reprobar la practica profesional y solicitar al estudiante que la repita, una vez que haya realizado las actividades remediales definidas.

Por otra parte, la dirección de la carrera deberá realizar un seguimiento a los resultados de las pruebas de logro, ya que si estas demuestran que alguna(s) competencia(s) no están siendo logradas de forma frecuente por los estudiantes, habrá que analizar como las asignaturas que dicen contribuir al desarrollo de la competencia(s) lo están haciendo.

\section{DISCUSIÓN}

La definición de la metodología propuesta, requirió buscar y combinar otros trabajos relacionados a los curriculum basado en competencias. En este aspecto la combinación de las propuestas de Tardiff (2003), Jacobs (2004) y Edward (2007) permitió establecer la metodología explicada en este artículo, pero hay que señalar que la propuesta de Edward (2007) quizás no sea útil a otras carreras, ya que su trabajo está enfocado en el área de la Ingenierías.

Al aplicar la metodología propuesta, se logró revisar y actualizar un proyecto educativo basado en competencias, obteniéndose mayor coherencia y consistencia en éste, ya que permitió encontrar y corregir desequilibrios en las asignaturas y el desarrollo de competencias y, establecer mecanismos de evaluación sobre el logro de éstas en los estudiantes. No obstante, se requiere que el cuerpo docente relacionado al proyecto educativo, se empoderé con la metodología propuesta, ya que de lo contrario, será compleja la aplicación de ésta. Además, y debido a que el Director de Carrera realiza otras funciones de gestión, es recomendable que no sea él quien lidere un proceso de revisión del curriculum, pero si que participe. Quizás un comité compuesto por algunos profesores sea mas adecuado para liderar este tipo de procesos.

Además es necesario fortalecer la metodología a través del uso de herramientas informáticas que integren toda la información utilizada en las diferentes etapas de la metodología, de manera que permitan simular diferentes escenarios sobre las asignaturas y el desarrollo de las competencias, así como también permitir el seguimiento del logro de éstas por los estudiantes. De esta manera se podrá evaluar si el proyecto educativo basado en competencias, está cumpliendo con los objetivos que se propuso.

Por último, la aplicación de la metodología propuesta, permitirá realizar un proceso de revisión y actualización de un proyecto educativo basado en competencia de manera mas eficiente y eficaz. 


\section{CONCLUSIONES}

De los resultados obtenido al aplicar la metodología para la revisión y actualización de un rediseño de currículo basado en competencias, de su análisis y de su discusión, se obtienen las siguientes conclusiones:

i) Contar con un proceso ordenado para el análisis del currículum permite generar evidencia suficiente para la toma de decisiones e involucrar a los profesores en generar intervenciones conducentes al logro del perfil de egreso esperado.

ii) El uso de herramientas gráficas de mapeo curricular genera productos comunicables que permiten discusiones con los docentes de manera que reenfoquen la tarea de enseñanza y evaluación en el marco de la visión de conjunto de la malla curricular.

iii) Debido a la cantidad de información generada en el proceso y los cambios que se producen entre los artefactos debido a la modificación y la relación entre ellos, se hace necesario contar con un sistema de información que permita gestionar el proceso como un todo y de esta manera, establecer distintos escenarios previos a la toma de decisiones.

\section{REFERENCIAS}

Argüelles, A. Competency based education and training: a world perspective. Editorial Limusa, (2000)

Beneitone, P., Esquetini, C., González, J., Marty, M., Slufi, G., y Wagenaar, R. Reflexiones y perspectivas de la educación superior en América Latina. Informe final. Proyecto Tuning América Latina 2004- 2007. Bilbao: Universidad de Deusto (2007)

Britton, M., Letassy, N., Medina, M. S., y Er, N. A curriculum review and mapping process supported by an electronic database system. American journal of pharmaceutical education , 72 (5) (2008)

Catalano, A., de Cols, S., y Sladogna, M. Competencia laboral: diseño curricular basado en normas de competencia laboral : conceptos y orientaciones metodológicas. Banco Interamericano de Desarrollo (2004)

CINDA. Diseño curricular basado en competencias y aseguramiento de la calidad en la educación superior. Centro Interuniversitario de desarrollo - CINDA (2009)

Corvalan, Ó. Perfil de competencias docentes y escalamiento para su desarrollo. Revista Electrónica de Desarrollo de Competencias , 1 (5), 72-105 (2010)

Edward, F., Johan, M., Soren, O., y Doris, B. Rethinking engineering education, the CDIO approach. Figure , 3, 67 (2007)

Fullan, M. Education in Motion: Leading in a culture of Change. Centre for Professional Excellence (2003)

Goñi, M., y Meseguer, S. Diseño curricular centrado en las competencias que debe adquirir el estudiante del grado en derecho. Formación universitaria , 3 (2), 37-46 (2010)

ISO 9000:2000, International Organization for Standarization. Sistemas de gestión de la calidad Requisitos. Suiza. (en línea) http://www.iso.org. Acceso 30 de Enero (2015)

Jacobs. Getting results with curriculum mapping. Alexandria, VA: Association for Supervision and Curriculum Development (2004)

Lemaitre, M. J., y Zenteno, M. E. Aseguramiento de la calidad en Iberoamérica. Educación Superior-Informe 2012 (2012)

Madrid, R. El derecho a la libertad de cátedra y el concepto de universidad. Revista chilena de derecho, 40(1), 355-371 (2013)

Schmal, R., y Ruiz-Tagle, A. Una metodología para el diseño de un currículo orientado a las competencias. Ingeniare. Revista chilena de ingeniería , 16 (1), 147-158 (2008)

Sudsomboon, W. Construction Of A Competency-Based Curriculum Content Framework For Mechanical Technology Education Program on Automotive Technology Subjects. Proceedings of the ICASE Asian Symposium (2007) 
Sutcliffe, N., Chan, S. S., y Nakayama, M. Competency Based MSIS Curriculum. Journal of Information Systems Education , 16 (3), 301 (2005)

Tardif, J. Développer un programme par compétences: de l'intention à la mise en oeuvre. Pédagogie collégiale , 16 (3), 36-44 (2003).

Tobón Tobón, S. Formación basada en Competencias, pensamiento complejo, diseño curricular y didáctica. Ecoe Ediciones. Bogotá (2004).

Vásquez Aqueveque, A. M., Apablaza Correa, R., Osorio Olivares, L., y Zuñiga Aguirre, J. Construcción en red de un currículo basado en competencias. Ciencia y enfermería, 17(3), 35-42 (2011) 